\title{
Recovery of functional connectivity of the sensorimotor network after surgery for diffuse low-grade gliomas involving the supplementary motor area
}

\author{
*Matthieu Vassal, MD, MSc, ${ }^{1-4}$ Céline Charroud, $\mathrm{PhD},{ }^{2,4}$ Jérémy Deverdun, $\mathrm{PhD},{ }^{2-6}$ \\ Emmanuelle Le Bars, PhD, ${ }^{2,4,6}$ François Molino, PhD, ${ }^{5,6}$ Francois Bonnetblanc, $\mathrm{PhD},{ }^{7}$ \\ Anthony Boyer, MSc, ${ }^{7}$ Anirban Dutta, PhD, ${ }^{7}$ Guillaume Herbet, PhD, ${ }^{1,3}$ Sylvie Moritz-Gasser, PhD, ${ }^{1,3}$ \\ Alain Bonafé, MD, PhD, ${ }^{2-4}$ Hugues Duffau, MD, PhD, ${ }^{1,3}$ and Nicolas Menjot de Champfleur, MD, PhD ${ }^{2-4,6}$

\begin{abstract}
Departments of ${ }^{1}$ Neurosurgery and ${ }^{4}$ Neuroradiology, ${ }^{2}$ Institut d'Imagerie Fonctionnelle Humaine, and ${ }^{3}$ Institut des Neurosciences de Montpellier, INSERM U1051, Centre Hospitalier Régional Universitaire de Montpellier; and 5 Institut de Génomique Fonctionnelle, UMR 5203-INSERM U661, 'Laboratoire Charles Coulomb, CNRS UMR 5221, and 7Laboratoire d'Informatique, de Robotique et de Microélectronique de Montpellier, CNRS UMR5506, Université de Montpellier, Montpellier, France
\end{abstract}

OBJECTIVE The supplementary motor area (SMA) syndrome is a well-studied lesional model of brain plasticity involving the sensorimotor network. Patients with diffuse low-grade gliomas in the SMA may exhibit this syndrome after resective surgery. They experience a temporary loss of motor function, which completely resolves within 3 months. The authors used functional MRI (fMRI) resting state analysis of the sensorimotor network to investigate large-scale brain plasticity between the immediate postoperative period and 3 months' follow-up.

METHODS Resting state fMRI was performed preoperatively, during the immediate postoperative period, and 3 months postoperatively in 6 patients with diffuse low-grade gliomas who underwent partial surgical excision of the SMA. Correlation analysis within the sensorimotor network was carried out on those 3 time points to study modifications of its functional connectivity.

RESULTS The results showed a large-scale reorganization of the sensorimotor network. Interhemispheric connectivity was decreased in the postoperative period, and increased again during the recovery process. Connectivity between the lesion side motor area and the contralateral SMA rose to higher values than in the preoperative period. Intrahemispheric connectivity was decreased during the immediate postoperative period and had returned to preoperative values at 3 months after surgery.

CONCLUSIONS These results confirm the findings reported in the existing literature on the plasticity of the SMA, showing large-scale modifications of the sensorimotor network, at both inter- and intrahemispheric levels. They suggest that interhemispheric connectivity might be a correlate of SMA syndrome recovery.

https://thejns.org/doi/abs/10.3171/2016.4.JNS152484

KEY WORDS magnetic resonance imaging; glioma; neuronal plasticity; paralysis; brain mapping; functional neuroimaging; oncology

$\mathrm{D}$ IFFUSE low-grade gliomas (DLGGs) are slowgrowing primitive brain tumors that occur predominantly in so-called functional regions. Because of their slow growth, they induce modifications of the local activity and connectivity, representing a wellstudied model of brain plasticity. Indeed, this plasticity is observed at different scales. 1) Some infiltrated regions continue to keep their functionality. 2) Functional regions migrate to the periphery of the tumor. 3) Finally, there might be a contralateral functional compensation. These plasticity phenomena are observable before, during, and after surgery, and are the topic of this study in the spe-

ABBREVIATIONS BOLD = blood-oxygen-level dependent; DLGG = diffuse low-grade glioma; fMRI = functional MRI; GRE-EPI = gradient echo-echo planar imaging; MNI = Montreal Neurological Institute; MP-RAGE = magnetization-prepared, rapid-acquisition gradient echo; SMA = supplementary motor area.

SUBMITTED October 28, 2015. ACCEPTED April 12, 2016.

INCLUDE WHEN CITING Published online June 17, 2016; DOI: 10.3171/2016.4.JNS152484.

* Drs. Vassal and Charroud contributed equally to this work. 
cific context of supplementary motor area (SMA) syndrome..$^{8,36}$

\section{Supplementary Motor Area Syndrome}

Described for the first time in 1977, 18,19 the SMA syndrome is characterized by a transitory akinesia of the hemibody contralateral to the damaged side. The SMA is organized somatotopically; thus, planning of a motor task would be managed by the pre-SMA, while the execution of the movement would be managed by the SMA, anteriorly for the upper limb, and further backward for the lower limb. ${ }^{14,16}$ SMA syndrome may also be associated with mutism when the lesion involves the dominant hemisphere..$^{13}$ It occurs postoperatively and resolves spontaneously and completely within weeks or months. ${ }^{4,9,29,30,38}$ There is a graduation in the intensity of SMA syndrome directly related to the somatotopic distribution of the lesion. For example, for a lesion occurring in the anterior part of the SMA, the lower limb motor function would be preserved. Brain plasticity associated with this syndrome remains poorly understood.

\section{Functional MRI and Brain Plasticity Studies}

Brain plasticity can be evaluated using different imaging techniques, ${ }^{15}$ in terms of either activation or connectivity.

Task functional MRI (fMRI) allows visualization of areas within the cortex that are activated by various stimuli or tasks. Using appropriate tasks and blood-oxygen-leveldependent (BOLD) signal, ${ }^{23}$ this imaging reveals cortical activations (resulting from metabolic and hemodynamic changes) associated with the task.

The BOLD signal reflects local neurovascular coupling and indirectly, local brain cortical activity. Thus, different regions of the brain are considered as working together when the temporal courses of their BOLD signals are correlated. Hence, using this technique, functional connectivity studies can focus on the concept of networks integrating different regions of the brain.

These regions can be either predetermined based on the literature (hypothesis-driven studies) or selected on the basis of a data-driven independent component analysis. ${ }^{22}$ The correlations in time between the associated time series are the basis of functional studies and allow investigation of coherent functional networks, as for example the sensorimotor network, in the brain considered as a dynamic system. The study of functional connectivity through intrinsic brain activity at rest seeks to identify the active brain areas during minimal brain activity, in the absence of any external stimulation. Several resting state networks have been identified in association with well-known functional structures (motor or language network). For other such networks, the precise role is still unknown. ${ }^{40}$

\section{The Sensorimotor Network}

As mentioned, SMA syndrome involves the motor network. This network is responsible for the initiation and execution of voluntary movements and comprises, from front to back: 1) a prefrontal area involved in movement planning, including the pre-SMA; 2) a frontal premotor area involved in movement coordination, corresponding to the premotor cortex (this area includes the SMA, which thus belongs to the frontomesial cortex, anterior to the primary motor cortex); 3) the primary motor cortex (i.e., precentral gyrus), from which the final movement command issues; and 4) the primary sensory cortex (i.e., postcentral gyrus), which allows adaptation through sensory feedback.

The motor network can be studied through fMRI motor tasks. However, this might not be possible in cases of acute deficit. In such cases, resting state MRI appears as a promising approach to identify the network and its reorganization. 14,39 The resting state sensorimotor network includes the SMA, the primary motor cortex, primary sensory cortex, insula, and cerebellum. This somatotopic distribution is spatially distributed identically in the resting state and the task-related motor network. 27,28,34

Analyzing functional connectivity of the sensorimotor network at rest seems to be the most promising means of studying its postoperative plasticity after DLGG resection.

\section{Postoperative Plasticity in the Context of Resection}

Plasticity of functional connectivity was observed with the use of resting state techniques in neurodegenerative, neurovascular, or neuropsychiatric diseases. Little is known about the reorganization of this connectivity in the aftermath of DLGG surgery.

Several magnetoencephalography studies found changes in functional connectivity of motor and language networks after resection of brain tumors. The networks exhibited changes in their preoperative functional connectivity that correlated with the observed neurocognitive deficits. ${ }^{5,32}$

The originality of this study is to identify the influence of DLGG resection on sensorimotor network's functional connectivity using resting state fMRI.

\section{Objectives and SMA Syndrome Biomarker Identification}

The objective of our study is to identify the reorganization functional connectivity of the sensorimotor network, using resting state fMRI, in patients with DLGG who exhibited SMA syndrome after surgery.

To investigate this plasticity, the network's functional connectivity is assessed 1) preoperatively, 2) in the immediate postoperative period while the patient is exhibiting SMA syndrome, and 3) at 3 months of follow-up, at a time when complete recovery is achieved.

In the more general context of biomarkers, our fMRI plasticity assessment aims at identifying specific noninvasive markers of the SMA syndrome, as tools to monitor its evolution and the recovery process. Due to the wholebrain spatial distribution of the network, such markers are expected to emerge only through a large-scale description of the plastic reorganization such as proposed here.

\section{Methods \\ Population}

Patients referred to our institution from 2012 to 2015 
were included in this study if they underwent awake resection of a histologically confirmed DLGG involving the SMA region.

Ethical committee of Montpellier gave its approval for this work. Informed consent was obtained from all individual participants for whom identifying information is included in this article.

\section{Clinical Evaluation}

A complete neurological examination was carried out preoperatively, in the immediate postoperative period, and at 3 months' follow-up, including manual muscle testing (as described in Daniels and Worthingham's Muscle Testing $^{11}$ ) of both upper and lower limbs. Motor testing was performed on the same day as each of the MRI examinations. Handedness was assessed using the Edinburgh Handedness Inventory. ${ }^{24}$

\section{Surgical Procedure}

Intraoperative mapping by direct cortical and subcortical electrical stimulation is the gold standard for detection and preservation of eloquent pathways during glioma surgery, because it allows for the performance of real-time anatomo-functional correlations. ${ }^{32,33}$ Awake electrical sensorimotor mapping at the cortical level was performed before tumor removal to determine the limits of resection. The posterior part of the SMA was systematically preserved to minimize the risk of deficits..$^{38}$ In addition, direct subcortical stimulation was performed during glioma removal to facilitate preservation of eloquent sensorimotor pathways.

\section{MRI Data Acquisition}

MRI examinations were performed at 3 different time points: 1) preoperatively, 2) 24 hours after surgery, and 3 ) 3 months after surgery. Images were acquired with a 1.5-T Avanto magnet or a 3-T Skyra magnet (Siemens); the same magnet was used for all 3 examinations for any given patient. All MRI examinations included resting state functional imaging as well as anatomical and diffusionweighted imaging.

\section{Resting State fMRI Acquisition}

Magnitude and phase images of the magnetic field were acquired with a gradient echo-echo planar imaging (GRE-EPI) sequence (TR $466 \mathrm{msec}$, TE1 $4.9 \mathrm{msec}$, TE2 $7.4 \mathrm{msec}$, voxel size $2.56 \times 2.56 \times 3 \mathrm{~mm}^{3}$, flip angle $60^{\circ}$ ).

Resting state fMRI acquisitions were performed using GRE-EPI (TR $2400 \mathrm{msec}$, TE $50 \mathrm{msec}, 200$ volumes, voxel size $3 \times 3 \times 5.5 \mathrm{~mm}^{3}$ ). During the resting state acquisition, the patients were instructed to keep their eyes closed and not to think of anything in particular.

\section{Anatomical Acquisition}

High-resolution anatomical images were acquired using a 3D magnetization-prepared, rapid-acquisition gradient echo (MP-RAGE) sequence (TR $1700 \mathrm{msec}$, TE 2.5 msec, TI $922 \mathrm{msec}$; flip angle $9^{\circ}$, voxel size $1 \times 1 \times 1 \mathrm{~mm}^{3}$ ) with 176 slices.

\section{MRI Data Analysis}

Morphological Analysis

Volumetric assessment of tumors on both preoperative and postoperative scans was performed manually, using the Myrian software platform (Myrian Expert VL, Intrasense; http://www.intrasense.fr).

\section{Functional Connectivity MRI Processing and Analysis.}

Given that the DLGGs were located within both hemispheres, we performed a right/left flip of resting state fMRI data within the group, to set all tumors in the right hemisphere, consequently labeled as the lesional hemisphere. The contralateral hemisphere is thus labeled as the healthy hemisphere.

\section{Resting State fMRI Data Preprocessing}

We used statistical parametric mapping 1 (SPM8, Wellcome Department of Imaging Neuroscience; http://www. fil.ion.ucl.ac.uk/spm/software/spm8/) as implemented in MATLAB (The MathWorks Inc.) for resting state image preprocessing.

It included corrections of magnetic field distortion, scan acquisition time difference, and head motion. Then a coregistration to the individual anatomical image was performed.

Resting state fMRI images were spatially normalized to Montreal Neurological Institute (MNI) space using a standard SPM8 template and then spatially smoothed using a Gaussian filter with a full width at half maximum of $6 \mathrm{~mm}$ to accommodate for interindividual anatomical variability.

Results are all expressed in the MNI space.

\section{Functional Connectivity Analysis}

Functional connectivity analysis was then performed in a 3-step process including 1) a spatial independent component analysis using the NetBrainWork software ${ }^{26,27}$ (https://sites.google.com/site/netbrainwork/, Laboratoire d'Imagerie Fonctionnelle), 2) a node selection for the sensorimotor network, and 3) a correlation analysis using the NetBrainWork software.

For each participant, the first 5 volumes were discarded to allow for equilibration of the magnetic field and the participants' adaptation to the scanning noise. ${ }^{35,37}$

\section{Visual Network as a Control Network}

To assess the specific plasticity of the motor network, we needed either a control group or an internal reference taken from another independent, clinically unimpaired functional network.

We selected the second option and assessed the plasticity of the visual network in each participant as a control, to differentiate incidental from specific effects of the surgical procedure on the motor network.

\section{Spatial Independent Component Analysis}

A spatial independent component analysis was performed for each participant independently, to visually identify among 30 components the one with a spatial distribution similar to that of the motor ${ }^{27}$ (Table 1). This component was determined based on a thresholding for a 
TABLE 1. Nodes constituting the sensorimotor network*

\begin{tabular}{|c|c|c|c|c|c|c|}
\hline \multirow[b]{3}{*}{ Region } & \multicolumn{6}{|c|}{ Coordinates } \\
\hline & \multicolumn{3}{|c|}{ Talairach Space } & \multicolumn{3}{|c|}{ MNI Space } \\
\hline & $x$ & $y$ & z & $x$ & y & z \\
\hline \multicolumn{7}{|l|}{ Frontal lobe } \\
\hline Rt SMA & 0 & -4 & 52 & 2 & 3 & 53 \\
\hline Lt SMA & 0 & -4 & 52 & 2 & 3 & 53 \\
\hline Rt primary motor cortex & 45 & -14 & 56 & 40 & -20 & 54 \\
\hline Lt primary motor cortex & -45 & -12 & 56 & -43 & -18 & 52 \\
\hline Rt rolandic operculum & 46 & -19 & 15 & 41 & -21 & 17 \\
\hline Lt rolandic operculum & -46 & -15 & 15 & -44 & -17 & 15 \\
\hline \multicolumn{7}{|l|}{ Cingulum } \\
\hline Rt ACC & 0 & 14 & 39 & -1 & 8 & 40 \\
\hline Lt ACC & 0 & 14 & 39 & -1 & 8 & 40 \\
\hline \multicolumn{7}{|l|}{ Parietal lobe } \\
\hline Rt postcentral gyrus & 53 & -11 & 38 & 48 & -16 & 38 \\
\hline Lt postcentral gyrus & -51 & -15 & 38 & -49 & -19 & 36 \\
\hline Rt SII & 55 & -30 & 22 & 50 & -32 & 22 \\
\hline Lt SII & -55 & -30 & 22 & -52 & -31 & 20 \\
\hline \multicolumn{7}{|l|}{ Insula } \\
\hline Rt posterior insula & 43 & -2 & 6 & 39 & -4 & 10 \\
\hline Lt posterior insula & -41 & 4 & 6 & -39 & 2 & 9 \\
\hline \multicolumn{7}{|l|}{ Cerebellum } \\
\hline Rt vermis VIII & 0 & -73 & -26 & -1 & -68 & -26 \\
\hline Lt vermis VIII & 0 & -73 & -26 & -1 & -68 & -26 \\
\hline
\end{tabular}

$\mathrm{ACC}=$ anterior cingular cortex; $\mathrm{SII}=$ secondary somatosensory cortex $($ parietal operculum).

* The selected regions are based on Perlbarg \& Marrelec, 2008. Coordinates are reported in Talairach and MNI spaces. significance value of $\mathrm{p}<0.01$ and a minimum cluster size of 5 voxels..$^{27}$ The same methodology was used to select the components of the visual network.

\section{Correlation Analysis}

Within the sensorimotor network and the visual network, a functional connectivity analysis was performed using the NetBrainWork software.

We applied the CORrection of Structured noise using spatial Independent Component Analysis (CORSICA) to remove the irrelevant fluctuations (head movements, physiological activity, and technical artifacts). ${ }^{26}$

Functional connectivity indices were based on correlations between 2 nodes using a Bayesian numerical sampling scheme. ${ }^{2,7,21}$ Finally, correlations within each node of the network were reported for each time point.

\section{Statistical Analysis}

To compare correlation coefficients between each time point, correlation coefficients were Fisher z-transformed.

Significant differences in correlations between pairs of nodes were assessed using a Kruskal-Wallis test for nonparametric continuous variables, on the Fisher ztransformed values from the entire group. All $\mathrm{p}$ values are 2 -sided, and a threshold of 0.05 was considered statistically significant. All analyses were performed using $\mathrm{R}$ software, version 3.0.2.

\section{Results}

\section{Population}

Six patients (1 male and 5 females), with a mean age of 38 years (SD 4.5 years), were included in this study (Table 2).

Five patients were right-handed and 1 was left-handed according to the Edinburgh Handedness Inventory. ${ }^{24}$

TABLE 2. Key patient characteristics*

\begin{tabular}{|c|c|c|c|c|c|c|}
\hline Characteristic & Patient 1 & Patient 2 & Patient 3 & Patient 4 & Patient 5 & Patient 6 \\
\hline Age & 42 & 43 & 32 & 39 & 33 & 37 \\
\hline Sex & $\mathrm{F}$ & $\mathrm{F}$ & $\mathrm{F}$ & $\mathrm{F}$ & $\mathrm{F}$ & $M$ \\
\hline Handedness & $\mathrm{Lt}$ & Rt & Rt & Rt & Rt & Rt \\
\hline Hemispheric side & $\mathrm{Rt}$ & $\mathrm{Lt}$ & $\mathrm{Rt}$ & $\mathrm{Rt}$ & $\mathrm{Lt}$ & Rt \\
\hline Tumor location & Frontal & Frontal & Frontal & $\begin{array}{l}\text { Fronto-insular \& } \\
\quad \text { callosal }\end{array}$ & Frontal & $\begin{array}{c}\text { Fronto-parieto- } \\
\text { cingular }\end{array}$ \\
\hline 1st symptom & Seizures & None & Headaches & Seizures & Seizures & Seizures \\
\hline \multicolumn{7}{|l|}{ Postop evaluation } \\
\hline Cognitive deficit & Yes & Yes & Yes & Yes & Yes & No \\
\hline Aphasia & No & Yes & No & No & Yes & No \\
\hline Motor facial deficit & No & No & No & No & Yes & Yes \\
\hline Motor upper-limb deficit & Yes & Yes & Yes & Yes & Yes & Yes \\
\hline Motor lower-limb deficit & No & No & No & No & No & No \\
\hline Follow-up (3 mos-recovery) & Yes & Yes & Yes & Yes & Yes & Yes \\
\hline Preop vol $\left(\mathrm{cm}^{3}\right)$ & 83 & 34 & 37 & 196 & 19 & 67 \\
\hline Postop vol $\left(\mathrm{cm}^{3}\right)$ & 49 & 2 & 0 & 65 & 7 & 49 \\
\hline Resection percentage (\%) & 41 & 94 & 100 & 67 & 63 & 27 \\
\hline
\end{tabular}

* Clinical characteristics of the 6 patients included in this study. All underwent awake resection of the DLGG, involving the SMA. 
Of the 6 tumors, 4 were located within the right hemisphere and 2 were located within the left hemisphere. They involved frontal regions and the SMA region (Fig. 1). The mean tumor volume was $72.7 \mathrm{~cm}^{3}\left(\mathrm{SD} 65.0 \mathrm{~cm}^{3}\right)$ preoperatively and $28.7 \mathrm{~cm}^{3}$ (SD $28.8 \mathrm{~cm}^{3}$ ) postoperatively. All tumors were histologically confirmed as WHO Grade II oligoastrocytomas.

\section{Clinical Evaluation}

Postoperatively, all patients experienced severe motor deficits of the upper limb, and 2 patients with left-hemisphere DLGGs also experienced mutism. No motor deficit of the lower limb was observed, as the posterior part of the SMA was systematically preserved. All patients had completely recovered by the end of the 3rd month after surgery, consistent with incomplete SMA syndrome.

Considering the time course of the clinical symptoms of SMA syndrome, the first MRI examination was performed before surgery, in the absence of symptoms; the second MRI examination was performed for all patients during the period of incomplete SMA syndrome; and the third MRI examination was performed after complete neurological recovery.

\section{Functional Connectivity}

Classes of interest representative of the sensorimotor network were obtained from the independent component analysis done on the whole group (Table 3 ).

Functional connectivity analysis of the sensorimotor network was based on 6 nodes preoperatively (left SMA, right postcentral gyrus, right precentral gyrus, left postcentral gyrus, left precentral gyrus, and right SMA), reduced to 5 nodes postoperatively due to right SMA resection.

Correlation coefficients, indicating strength of connection between the different nodes, were calculated for each time point (preoperative, postoperative, and 3 months after surgery), between the different nodes (left SMA, right postcentral gyrus, right precentral gyrus, left postcentral gyrus, left precentral gyrus, and right SMA for the preoperative condition) (Figs. 2 and 3, Table 4).

With regard to interhemispheric connectivity, the Kruskal-Wallis test, performed on z-transformed correlation coefficients, found statistically significant differences $(\mathrm{p}=$ 0.020 ) between the 3 time points (preoperative, $z=0.043$; postoperative, $\mathrm{z}=-0.094$; and follow-up, $\mathrm{z}=0.032$ ), immediate postoperative correlations being lower than preoperative and follow-up values. As mentioned above, we used the visual network as a control to assess the significance of this postoperative decrease in interhemispheric connectivity. We found a paradoxical increase in interhemispheric connectivity postoperatively in the visual network ( $p=0.02$; preoperative, $\mathrm{z}=0.31$; postoperative, $\mathrm{z}=0.55$; and follow-up, $\mathrm{z}=0.29$ ), in the absence of any visual symptoms. This result, which may merit further investigation, strongly suggests in any case that the decrease observed in the motor network is not an incidental consequence of the surgical procedure.

Exploring intrahemispheric connectivity, using the same procedure, the Kruskal-Wallis test, performed on

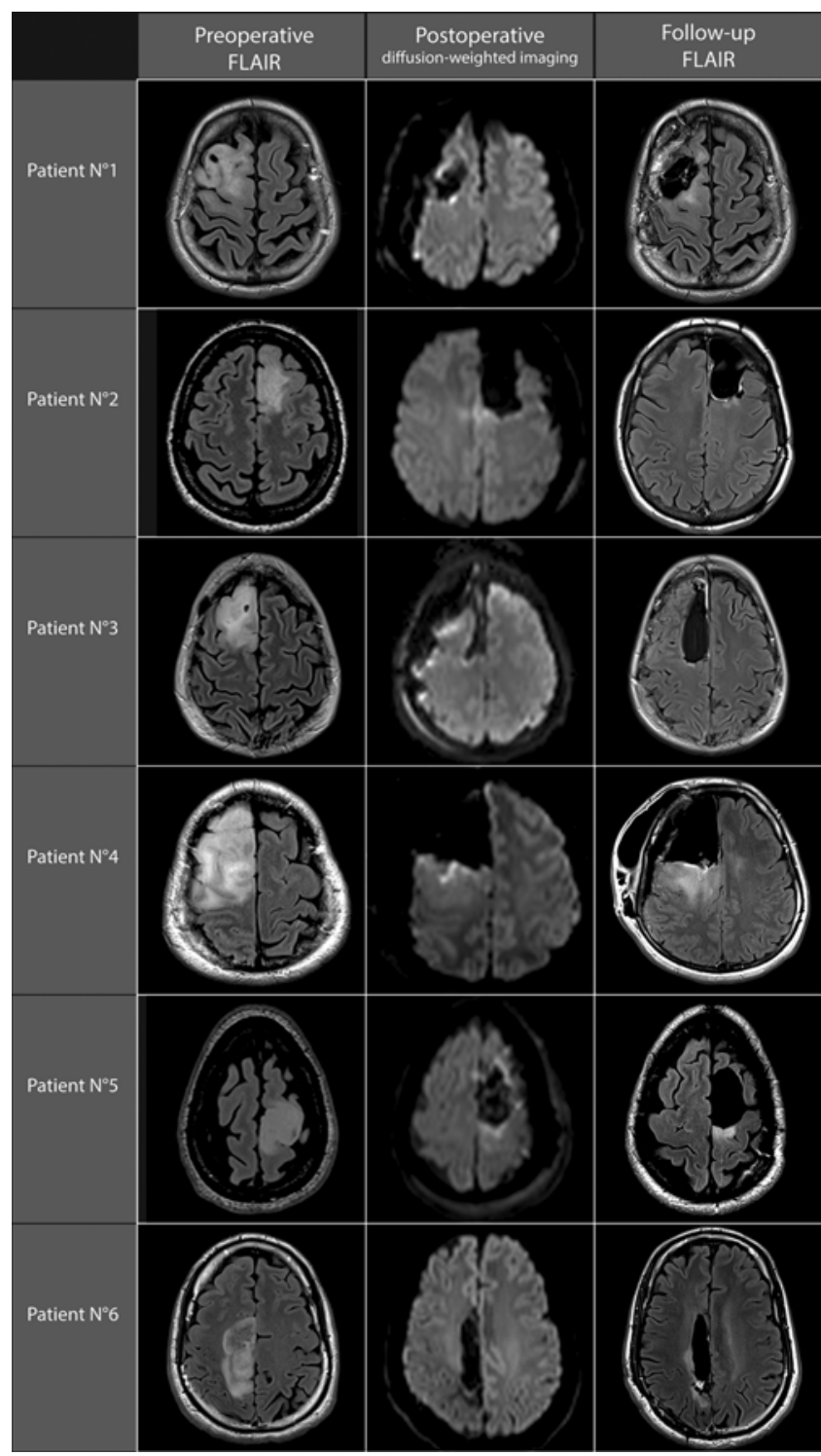

FIG. 1. Anatomical and diffusion-weighted imaging. Each row includes images obtained in an individual patient. The left column of images displays preoperative FLAIR acquisitions; the center column, diffusionweighted images acquired in the postoperative period to rule out any ischemic lesion; the right column, FLAIR images obtained in the followup period.

z-transformed correlation coefficients, failed to find any statistically significant difference $(p=0.19)$ between the 3 time points (preoperative, $\mathrm{z}=0.025$; postoperative, $\mathrm{z}=$ -0.12 ; and follow-up, $\mathrm{z}=0.030$ ).

\section{Discussion}

The principal aim of this study was to identify, using resting state fMRI, large-scale reorganizations in the sensorimotor network specifically associated with SMA syndrome. We observed that reorganization occurs at 2 different scales. 1) The postoperative period is characterized by a significant decrease in global interhemispheric correlations, followed at 3 months after surgery by a return 
TABLE 3. Coordinates of sensorimotor network nodes as derived from the independent component analysis of resting state fMRI data*

\begin{tabular}{|c|c|c|c|c|c|c|c|c|c|c|c|c|c|c|c|c|c|c|}
\hline \multirow[b]{3}{*}{ Node } & \multicolumn{6}{|c|}{ Preop } & \multicolumn{6}{|c|}{ Postop } & \multicolumn{6}{|c|}{ Follow-Up } \\
\hline & \multicolumn{3}{|c|}{ Talairach Space } & \multicolumn{3}{|c|}{ MNI Space } & \multicolumn{3}{|c|}{ Talairach Space } & \multicolumn{3}{|c|}{ MNI Space } & \multicolumn{3}{|c|}{ Talairach Space } & \multicolumn{3}{|c|}{ MNI Space } \\
\hline & $x$ & $\mathrm{y}$ & z & $x$ & $\mathrm{y}$ & z & $x$ & $\mathrm{y}$ & z & $\mathrm{x}$ & $\mathrm{y}$ & z & $x$ & $y$ & z & $\mathrm{x}$ & $\mathrm{y}$ & z \\
\hline Rt SMA & 7 & -1 & 52 & 9 & 6 & 53 & NA & NA & NA & NA & NA & NA & NA & NA & NA & NA & NA & NA \\
\hline Lt SMA & -1 & -7 & 55 & 1 & 0 & 57 & -1 & -1 & 59 & 1 & 7 & 61 & 0 & -3 & 55 & 2 & 4 & 57 \\
\hline Rt precentral gyrus & 60 & 0 & 32 & 66 & 5 & 30 & 42 & -3 & 49 & 47 & 4 & 49 & 34 & -6 & 44 & 38 & 0 & 44 \\
\hline Lt precentral gyrus & -55 & 11 & 30 & -58 & 16 & 28 & -20 & -18 & 62 & -20 & -11 & 66 & -56 & 10 & 23 & -59 & 14 & 21 \\
\hline Rt postcentral gyrus & 37 & -26 & 59 & 42 & -20 & 63 & 27 & -24 & 60 & 31 & -18 & 64 & 31 & -30 & 56 & 35 & -25 & 60 \\
\hline Lt postcentral gyrus & -34 & -26 & 62 & -35 & -20 & 67 & -47 & -11 & 26 & -49 & -8 & 26 & -40 & -34 & 62 & -41 & -28 & 68 \\
\hline
\end{tabular}

NA = not applicable.

* Coordinates of the sensorimotor network nodes derived from the independent component analysis of the resting state, for each time point of the study. Coordinates are reported in Talairach and MNI spaces. For the lesional hemisphere SMA, coordinates are not reported in the postoperative period because of its resection.

to the preoperative values. Indeed the correlation between the healthy-side SMA and the lesion-side sensorimotor network is increased compared with the preoperative values. 2) With respect to intrahemispheric correlations, we observed postoperative decreases in correlations on the lesion side, which is a logical consequence of the resection of the SMA. Three months after surgery, the intrahemispheric correlations returned to preoperative values.

\section{Plasticity of the Functional Connectivity}

Previous studies using task fMRI as well as resting state data have suggested plasticity phenomena both around the tumor and in terms of large-scale reorganization.

Using a task fMRI protocol, we found increased activations within the healthy-side SMA after DLGG resection..$^{15}$ In patients with clinical motor impairment after brain tumor resection, decreased postoperative activations in the primary motor areas ipsilateral to the tumor have been observed simultaneously with increased activations of the ispilateral secondary motor areas (SMA and superior parietal lobule).$^{28}$

Task fMRI cannot be performed in severely impaired patients, requiring the use of an alternative approach such as resting state fMRI with observation of the sensorimotor network through correlation analysis. ${ }^{26}$ Although it is well known that the results of resting state analysis can be modulated by attention or emotional state, the robustness of such analyses is nevertheless remarkable, ${ }^{3,6}$ particularly when comparing several MRI examinations in a longitudinal study. ${ }^{31}$

Various studies have demonstrated the effectiveness of this approach in investigating brain plasticity. With respect to skill learning, studies have shown that functional connectivity of the sensorimotor network increases with coordination learning. ${ }^{20}$ With regard to neurovascular diseases, large-scale plasticity phenomena have been described in

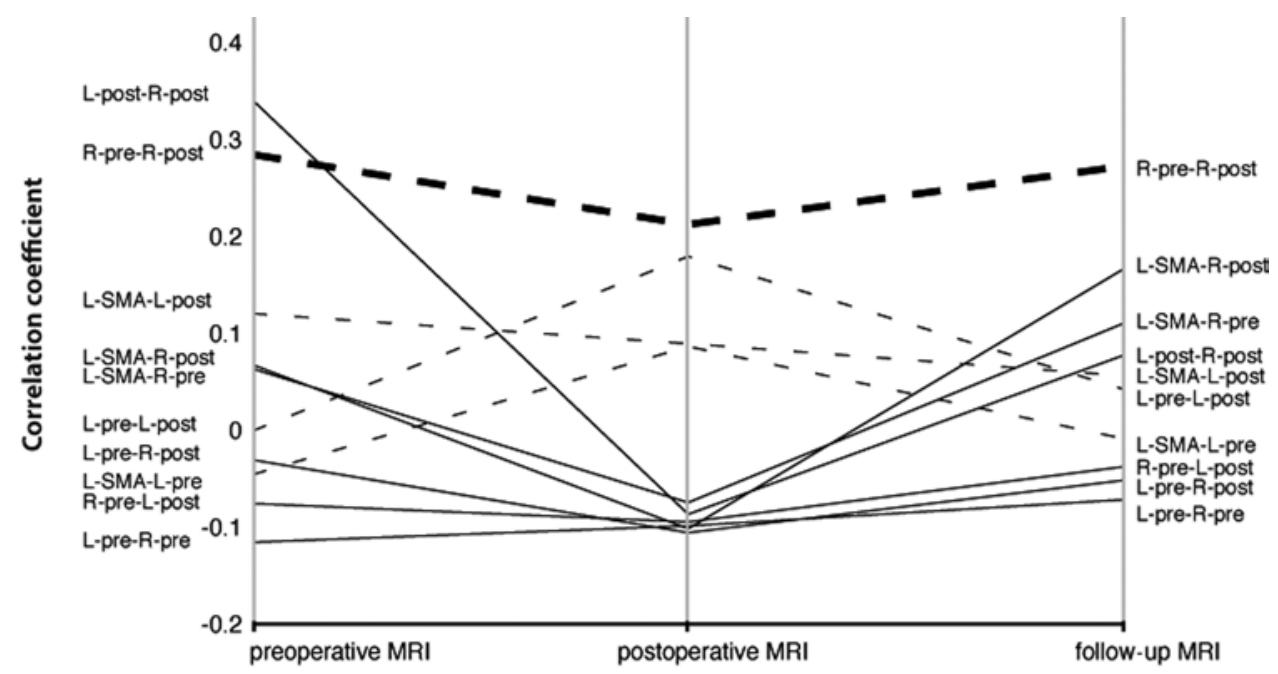

FIG. 2. Longitudinal evolution plot of inter- and intrahemispheric correlations in the sensorimotor network. The time course of all intrahemispheric (dashed lines) and interhemispheric (continuous lines) correlation coefficients within the sensorimotor network are presented. For purposes of this study, right $(R)$ is always the lesional hemisphere, and left $(L)$ the healthy hemisphere. The temporary decreases in interhemispheric correlations between the healthy-side SMA and the contralateral motor areas and between the 2 postcentral regions are statistically significant (Kruskal-Wallis test performed on z-transformed correlation coefficients). post = postcentral gyrus; pre = precentral gyrus. 
Intrahemispheric correlations

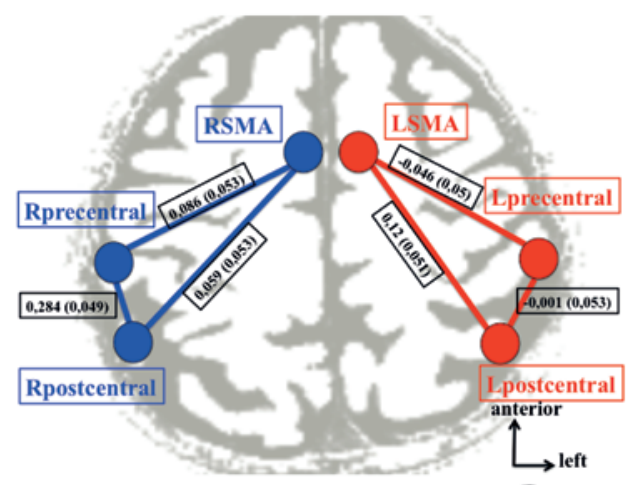

\section{Preoperative}
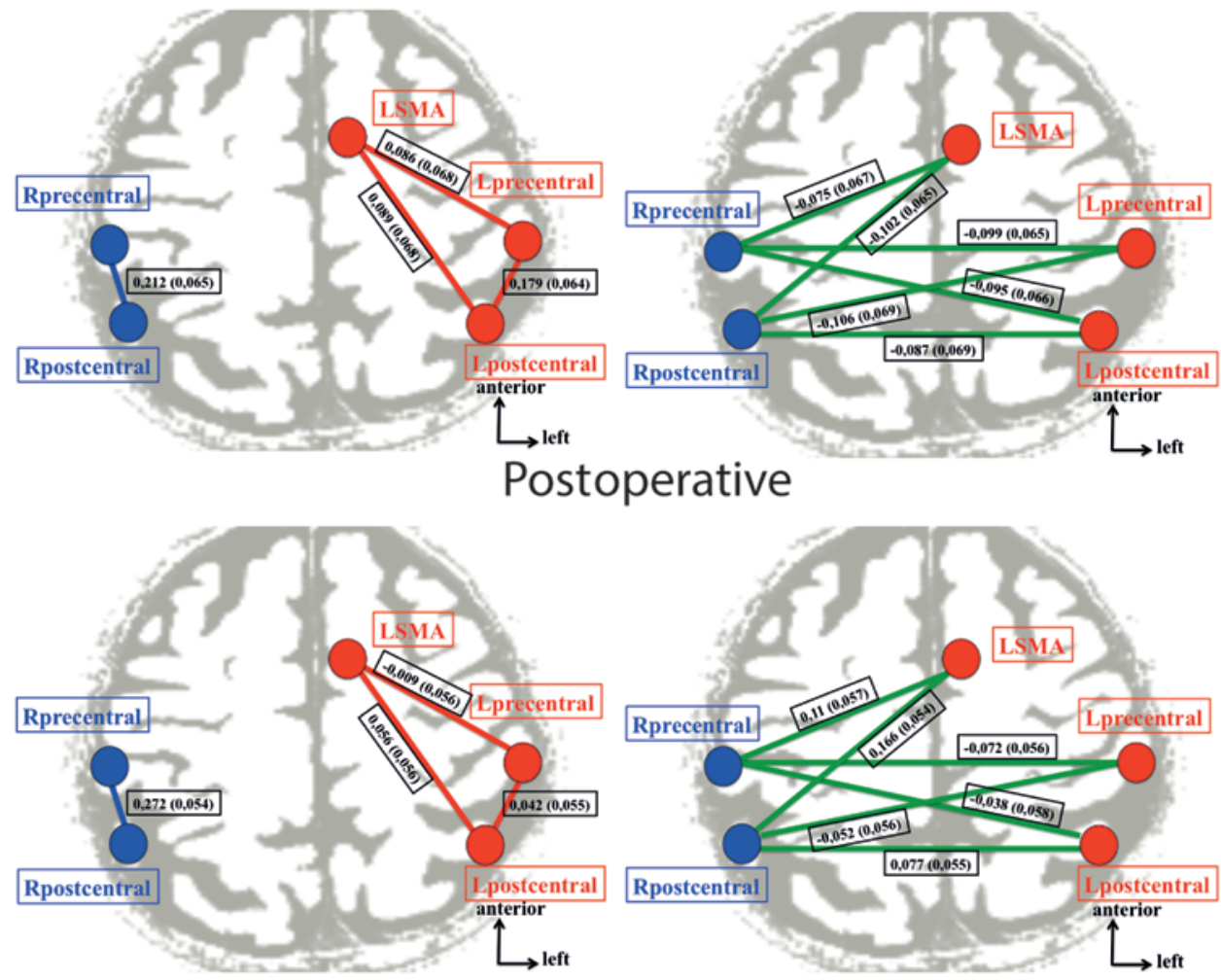

\section{Follow-up}

FIG. 3. Evolution of correlation maps within the resting state sensorimotor network. Correlation coefficients between the sensorimotor network nodes, at both intrahemispheric (left column) and interhemispheric (right column) levels, in the preoperative period (top), in the immediate postoperative period (center), and at 3 months' follow-up (bottom). In both hemispheres, network nodes include the precentral region, the postcentral region, and the SMA; the SMA was resected on the lesional side and is therefore absent postoperatively. Each correlation is presented with its associated standard deviation. Note that in these data the right hemisphere is always the lesional hemisphere, the left hemisphere being the healthy hemisphere. Figure is available in color online only.

stroke, involving the sensorimotor network. In particular, correlations between motor rehabilitation and interhemispheric connectivity have been observed, with decreased interhemispheric connectivity followed by a return to normal values concomitant with motor recovery. ${ }^{2,11,12}$

Finally, in the specific pathological context of DLGG, it is also noteworthy that preoperative motor deficits have been associated with decreased interhemispheric correlations in the sensorimotor network..$^{25}$

\section{Plasticity of Interhemispheric Functional Connectivity}

Using a similar resting state fMRI approach, we 
TABLE 4. Within-system correlations between nodes of the sensorimotor network for the 3 time points*

\begin{tabular}{|c|c|c|c|c|c|c|c|c|c|c|}
\hline \multirow{2}{*}{\multicolumn{2}{|c|}{ Node 1}} & \multirow{2}{*}{\multicolumn{2}{|c|}{ Node 2}} & \multicolumn{6}{|c|}{ Time Point } & \multirow{3}{*}{$\begin{array}{c}p \\
\text { Valuet }\end{array}$} \\
\hline & & & & \multicolumn{2}{|c|}{ Preop } & \multicolumn{2}{|c|}{ Postop } & \multicolumn{2}{|c|}{ Follow-Up } & \\
\hline Side & Topography & Side & Topography & r & SD & $r$ & SD & r & SD & \\
\hline Interhemispheric connectivity & & & & & & & & & & 0.0195 \\
\hline \multirow[t]{2}{*}{ Lt } & SMA & Rt & Postcentral gyrus & 0.067 & 0.051 & -0.102 & 0.065 & 0.166 & 0.054 & \\
\hline & & Rt & Precentral gyrus & 0.063 & 0.051 & -0.075 & 0.067 & 0.11 & 0.057 & \\
\hline \multirow[t]{2}{*}{$\mathrm{Lt}$} & Precentral gyrus & $\mathrm{Rt}$ & Precentral gyrus & -0.116 & 0.051 & -0.099 & 0.065 & -0.072 & 0.056 & \\
\hline & & Rt & Postcentral gyrus & -0.031 & 0.054 & -0.106 & 0.069 & -0.052 & 0.056 & \\
\hline \multirow[t]{2}{*}{ Lt } & Postcentral gyrus & Rt & Precentral gyrus & -0.076 & 0.053 & -0.095 & 0.066 & -0.038 & 0.058 & \\
\hline & & Rt & Postcentral gyrus & 0.34 & 0.052 & -0.087 & 0.069 & 0.077 & 0.055 & \\
\hline Intrahemispheric connectivity & & & & & & & & & & 0.1931 \\
\hline \multirow[t]{2}{*}{ Lt } & SMA & $\mathrm{Lt}$ & Precentral gyrus & -0.046 & 0.05 & 0.086 & 0.068 & -0.009 & 0.056 & \\
\hline & & $\mathrm{Lt}$ & Postcentral gyrus & 0.012 & 0.051 & 0.089 & 0.068 & 0.056 & 0.056 & \\
\hline Lt & Precentral gyrus & $\mathrm{Lt}$ & Postcentral gyrus & -0.001 & 0.053 & 0.179 & 0.064 & 0.042 & 0.055 & \\
\hline Rt & Precentral gyrus & Rt & Postcentral gyrus & 0.284 & 0.049 & 0.212 & 0.065 & 0.272 & 0.054 & \\
\hline
\end{tabular}

$r=$ correlation coefficient; SD = standard deviation.

* With respect to interhemispheric connectivity, the Kruskal-Wallis test, done on z-transformed correlation coefficients, found statistically significant differences ( $p=$ 0.01953 ) between the 3 time points (preoperative, $z=0.04343$; postoperative, $z=-0.09429$; and follow-up, $z=0.03216$ ), immediate postoperative correlations being lower than preoperative and follow-up values. Exploring intrahemispheric connectivity using the same procedure, the Kruskal-Wallis test, done on z-transformed correlation coefficients, failed to find any statistically significant difference $(p=0.1931)$ between the 3 time points (preoperative, $z=0.02452$; postoperative, $z=-0.11880$; and follow-up, $z=0.02969$ ). Note that the right hemisphere is the lesional hemisphere, the left hemisphere being the healthy hemisphere.

$\dagger$ Kruskal-Wallis test.

identified large-scale reorganizations of the sensorimotor network after surgery, which can be associated with SMA syndrome. In the postoperative period we identified significant decreases in interhemispheric correlations, followed by a return to the preoperative values at 3 months after surgery, when SMA syndrome had resolved. This result regarding interhemispheric connectivity is in agreement with findings of previously mentioned stroke studies. $^{2}$

In relation to previous studies, ${ }^{25}$ it is very interesting to note also that interhemispheric connectivity is now demonstrated to be both inversely correlated to preoperative deficit and positively correlated with postoperative recovery in SMA syndrome.

Finally, with regard to interhemispheric connectivity between the primary sensorimotor cortex ipsilateral to the tumor and the contralateral SMA, our results showed an increase in this specific connectivity simultaneous with motor recovery. This suggests that the healthy-side SMA could assume a new role in the sensorimotor network, as a substitute for the resected SMA. This postoperative reorganization could help motor deficit recovery. It corroborates previous observations, emphasizing the role of preoperative contralateral reorganization of the network, sustaining rapid motor recovery. ${ }^{15,17,34}$

\section{Plasticity of Intrahemispheric Functional Connectivity}

Regarding intrahemispheric connectivity, we observed a postoperative decrease of functional connectivity within the operated hemisphere, which might be due to the resection itself. In the contralateral hemisphere, we noted a slight (nonsignificant) increase in connectivity, between the primary motor area and the ipsilateral SMA. After 3 months of follow-up, the intrahemispheric correlations returned to their preoperative values.

\section{Perspectives}

The identification through fMRI of plastic changes in the brain specific to a given syndrome (e.g., SMA syndrome) must be put in the perspective of a more global strategy: identification of noninvasive markers of brain plasticity. These results support a potential role for interhemispheric functional connectivity as a marker of motor recovery in the specific context of SMA syndrome.

\section{Conclusions}

To our knowledge, this is the first resting state fMRI study to identify perioperative changes in functional connectivity in patients with DLGG. Previous studies have demonstrated large-scale postoperative reorganization of motor activations, concomitant with neurological recovery. Our results are consistent with those previous observations and contribute to emphasize the large-scale plasticity of functional connectivity. The usual description, according to which the healthy-side SMA assumes the role of the resected one during recovery, remains correct but fails to capture the large-scale plastic reorganization of the network and particularly its interhemispheric component.

\section{References}

1. Ashburner J: SPM: a history. Neuroimage 62:791-800, 2012

2. Biswal B, Yetkin FZ, Haughton VM, Hyde JS: Functional connectivity in the motor cortex of resting human brain using echo-planar MRI. Magn Reson Med 34:537-541, 1995 
3. Biswal BB, Mennes M, Zuo XN, Gohel S, Kelly C, Smith SM, et al: Toward discovery science of human brain function. Proc Natl Acad Sci U S A 107:4734-4739, 2010

4. Bleasel A, Comair Y, Lüders HO: Surgical ablations of the mesial frontal lobe in humans. Adv Neurol 70:217-235, 1996

5. Bosma I, Stam CJ, Douw L, Bartolomei F, Heimans JJ, van Dijk BW, et al: The influence of low-grade glioma on resting state oscillatory brain activity: a magnetoencephalography study. J Neurooncol 88:77-85, 2008

6. Chou YH, Panych LP, Dickey CC, Petrella JR, Chen NK: Investigation of long-term reproducibility of intrinsic connectivity network mapping: a resting-state fMRI study. AJNR Am J Neuroradiol 33:833-838, 2012

7. Coynel D, Marrelec G, Perlbarg V, Pélégrini-Issac M, Van de Moortele PF, Ugurbil K, et al: Dynamics of motor-related functional integration during motor sequence learning. Neuroimage 49:759-766, 2010

8. Duffau H, Capelle L, Denvil D, Gatignol P, Sichez N, Lopes $\mathrm{M}$, et al: The role of dominant premotor cortex in language: a study using intraoperative functional mapping in awake patients. Neuroimage 20:1903-1914, 2003

9. Duffau H, Lopes M, Denvil D, Capelle L: Delayed onset of the supplementary motor area syndrome after surgical resection of the mesial frontal lobe: a time course study using intraoperative mapping in an awake patient. Stereotact Funct Neurosurg 76:74-82, 2001

10. Golestani AM, Tymchuk S, Demchuk A, Goodyear BG: Longitudinal evaluation of resting-state FMRI after acute stroke with hemiparesis. Neurorehabil Neural Repair 27:153-163, 2013

11. Hislop H, Avers D, Brown M: Daniels and Worthingham's Muscle Testing, ed 9. St. Louis: Elsevier Saunders, 2014

12. James GA, Lu ZL, VanMeter JW, Sathian K, Hu XP, Butler AJ: Changes in resting state effective connectivity in the motor network following rehabilitation of upper extremity poststroke paresis. Top Stroke Rehabil 16:270-281, 2009

13. Kinoshita M, de Champfleur NM, Deverdun J, MoritzGasser S, Herbet G, Duffau H: Role of fronto-striatal tract and frontal aslant tract in movement and speech: an axonal mapping study. Brain Struct Funct 220:3399-3412, 2015

14. Kokkonen SM, Nikkinen J, Remes J, Kantola J, Starck T, Haapea M, et al: Preoperative localization of the sensorimotor area using independent component analysis of resting-state fMRI. Magn Reson Imaging 27:733-740, 2009

15. Krainik A, Duffau H, Capelle L, Cornu P, Boch AL, Mangin JF, et al: Role of the healthy hemisphere in recovery after resection of the supplementary motor area. Neurology 62:1323-1332, 2004

16. Krainik A, Lehéricy S, Duffau H, Capelle L, Chainay H, Cornu P, et al: Postoperative speech disorder after medial frontal surgery: role of the supplementary motor area. Neurology 60:587-594, 2003

17. Krainik A, Lehéricy S, Duffau H, Vlaicu M, Poupon F, Capelle L, et al: Role of the supplementary motor area in motor deficit following medial frontal lobe surgery. Neurology 57:871-878, 2001

18. Laplane D, Talairach J, Meininger V, Bancaud J, Bouchareine A: Motor consequences of motor area ablations in man. $\mathbf{J}$ Neurol Sci 31:29-49, 1977

19. Laplane D, Talairach J, Meininger V, Bancaud J, Orgogozo JM: Clinical consequences of corticectomies involving the supplementary motor area in man. J Neurol Sci 34:301-314, 1977

20. Ma L, Narayana S, Robin DA, Fox PT, Xiong J: Changes occur in resting state network of motor system during 4 weeks of motor skill learning. Neuroimage 58:226-233, 2011

21. Marrelec G, Krainik A, Duffau H, Pélégrini-Issac M, Lehéricy S, Doyon J, et al: Partial correlation for functional brain interactivity investigation in functional MRI. Neuroimage 32:228-237, 2006

22. Meyer MC, van Oort ES, Barth M: Electrophysiological correlation patterns of resting state networks in single subjects: a combined EEG-fMRI study. Brain Topogr 26:98-109, 2013

23. Ogawa S, Menon RS, Tank DW, Kim SG, Merkle H, Ellermann JM, et al: Functional brain mapping by blood oxygenation level-dependent contrast magnetic resonance imaging. A comparison of signal characteristics with a biophysical model. Biophys J 64:803-812, 1993

24. Oldfield RC: The assessment and analysis of handedness: the Edinburgh inventory. Neuropsychologia 9:97-113, 1971

25. Otten ML, Mikell CB, Youngerman BE, Liston C, Sisti MB, Bruce JN, et al: Motor deficits correlate with resting state motor network connectivity in patients with brain tumours. Brain 135:1017-1026, 2012

26. Perlbarg V, Bellec P, Anton JL, Pélégrini-Issac M, Doyon J, Benali H: CORSICA: correction of structured noise in fMRI by automatic identification of ICA components. Magn Reson Imaging 25:35-46, 2007

27. Perlbarg V, Marrelec G: Contribution of exploratory methods to the investigation of extended large-scale brain networks in functional MRI: methodologies, results, and challenges. Int J Biomed Imaging 2008:218519, 2008

28. Reinges MH, Krings T, Rohde V, Hans FJ, Willmes K, Thron A, et al: Prospective demonstration of short-term motor plasticity following acquired central pareses. Neuroimage 24:1248-1255, 2005

29. Rostomily RC, Berger MS, Ojemann GA, Lettich E: Postoperative deficits and functional recovery following removal of tumors involving the dominant hemisphere supplementary motor area. J Neurosurg 75:62-68, 1991

30. Russell SM, Kelly PJ: Incidence and clinical evolution of postoperative deficits after volumetric stereotactic resection of glial neoplasms involving the supplementary motor area. Neurosurgery 52:506-516, 2003

31. Shehzad Z, Kelly AM, Reiss PT, Gee DG, Gotimer K, Uddin LQ, et al: The resting brain: unconstrained yet reliable. Cereb Cortex 19:2209-2229, 2009

32. Tarapore PE, Tate MC, Findlay AM, Honma SM, Mizuiri D, Berger MS, et al: Preoperative multimodal motor mapping: a comparison of magnetoencephalography imaging, navigated transcranial magnetic stimulation, and direct cortical stimulation. J Neurosurg 117:354-362, 2012

33. Tate MC, Herbet G, Moritz-Gasser S, Tate JE, Duffau H: Probabilistic map of critical functional regions of the human cerebral cortex: Broca's area revisited. Brain 137:27732782,2014

34. van den Heuvel OA, Remijnse PL, Mataix-Cols D, Vrenken $\mathrm{H}$, Groenewegen HJ, Uylings HB, et al: The major symptom dimensions of obsessive-compulsive disorder are mediated by partially distinct neural systems. Brain 132:853-868, 2009

35. Wang L, Zang Y, He Y, Liang M, Zhang X, Tian L, et al: Changes in hippocampal connectivity in the early stages of Alzheimer's disease: evidence from resting state fMRI. Neuroimage 31:496-504, 2006

36. Weiller C, Rijntjes M: Learning, plasticity, and recovery in the central nervous system. Exp Brain Res 128:134-138, 1999

37. Wu X, Li R, Fleisher AS, Reiman EM, Guan X, Zhang $Y$, et al: Altered default mode network connectivity in Alzheimer's disease - a resting functional MRI and Bayesian network study. Hum Brain Mapp 32:1868-1881, 2011

38. Zentner J, Hufnagel A, Pechstein U, Wolf HK, Schramm J: Functional results after resective procedures involving the supplementary motor area. J Neurosurg 85:542-549, 1996

39. Zhang D, Johnston JM, Fox MD, Leuthardt EC, Grubb RL, 
Chicoine MR, et al: Preoperative sensorimotor mapping in brain tumor patients using spontaneous fluctuations in neuronal activity imaged with functional magnetic resonance imaging: initial experience. Neurosurgery 65 (6 Suppl):226-236, 2009

40. Zhang X, Li X, Jin C, Chen H, Li K, Zhu D, et al: Identifying and characterizing resting state networks in temporally dynamic functional connectomes. Brain Topogr 27:747-765, 2014

\section{Disclosures}

The authors report no conflict of interest concerning the materials or methods used in this study or the findings specified in this paper.

\section{Author Contributions}

Acquisition of data: Vassal, Herbet, Moritz-Gasser, Duffau. Analysis and interpretation of data: Deverdun, Vassal, Charroud, Le Bars, Menjot de Champfleur. Drafting the article: Vassal, Molino, Menjot de Champfleur. Critically revising the article: Deverdun, Vassal, Le Bars, Bonnetblanc, Boyer, Dutta, Herbet, Moritz-Gasser, Bonafé, Duffau, Menjot de Champfleur. Reviewed submitted version of manuscript: Deverdun, Vassal. Statistical analysis: Vassal.

\section{Correspondence}

Jérémy Deverdun, Department of Neuroradiology, University Hospital Center, Gui de Chauliac Hospital, 80 Ave. Augustin Fliche, 34295 Montpellier Cedex 5, France. email: jeremy. deverdun@neuf.fr. 